\title{
Periodontal status of type I diabetics compared to non-diabetic participants: a preliminary study
}

\author{
S P Kakade, S H Shetiya, P Kakodkar, R V Shirahatti, D Agrawal
}

(Index words: diabetes mellitus, type I; periodontitis; oral health)

\begin{abstract}
Summary
A preliminary group matched comparison study of periodontal status was conducted among Indian participants who had type 1 diabetes mellitus (T1DM) and non-diabetic controls. The mean number of bleeding sites, gigival index score, number of periodontal pocket's and number of clinical loss attachment sites were all significantly higher in cases compared to controls. This study provides evidence that people with type I diabetes mellitus have a higher risk of gingival and periodontal disease.
\end{abstract}

Ceylon Medical Journal 2014; 59: 19-20

\section{Introduction}

Periodontitis is one of the complications of type II diabetes mellitus but the association with type 1 diabetes mellitus (T1DM) is less clear $[1,2,3]$. Although patients with diabetes mellitus may have an increased susceptibility to periodontal disease, there is little data on prevalence of periodontal disease in patients with T1DM, especially in younger age groups. This study was planned to compare periodontal status of T1DM and non-diabetic individuals in Pune city, India.

\section{Methods}

A preliminary group matched comparison study was conducted and cases with (type I diabetes mellitus) [4] were recruited from a private hospital in Pune. Age and sex matched controls (non-diabetic healthy participants) were selected using convenience sampling. Inclusion criteria were a diagnosis of T1DM on insulin therapy for more than 12 months, an HbA1c level more than 7\%, and age between 15 and 35 years. For each case, two controls were recruited. A pilot study was conducted and an interim analysis was carried out to calculate sample size.

A validated performa was designed to record the relevant demographic and clinical information. Inter rater reliability was assessed using ten non-diabetic participants aged 15-35 years with early to moderately advanced periodontitis. The inter-rater reliability was 0.9 (kappa value). The data were collected by a single examiner from 6th August to 1st October 2011.

Before commencing the study approval was taken from the local Scientific Board and Ethical Committee. Informed consent was obtained from participants; consent forms were prepared both in English and Marathi (Local Language).

At the first appointment a detailed history of the participants with T1DM was obtained and the blood glucose level was assessed using Accu-chek blood glucose meter. Oral examination was done and gingival index was used to assess severity of gingivitis [5]. Bleeding on probing, clinical attachment loss (LOA) and periodontal pockets were recorded using Community Periodontal Index (CPI) probe for all teeth as per World Health Organization Oral Health Assessment Form 1997 [6]. A detailed history was also obtained from non-diabetic healthy participants and the blood glucose level was recorded.

Data were entered into Microsoft Office Excel 2007 and analysed using Statistical Package for Social Sciences (SPSS) Inc. version 17.0 Chicago, USA. Onesample Kolmogorov-Smirnov test was used to explore the normalcy of the data. Mann Whitney $\mathrm{U}$ test and independent sample t-Test was used to assess the difference in means between the groups.

\section{Results}

We studied 66 participants [ 22 cases and 44 controls; mean age (SD) 20.95 (5.34) years]. The demographic and periodontal characteristics of the study participants are shown in the Table. The mean time duration of T1DM was 9.86 (SD 5.77) years. The mean number of bleeding sites was 12.32 in cases and 3.4 in the controls $(p<0.05)$. The mean number of periodontal pockets was 2.23 in cases and 1.32 in controls $(p<0.05)$. The mean number of sites of loss of clinical attachment was 2.14 in cases and 1.57 in controls ( $p>0.05$ ). The mean gingival index score was 1.40 and 0.92 in cases and controls $(p<0.05)$ respectively [CI $95 \%-0.28$ to 0.67$]$.

Department of Public Health Dentistry, Dr. D. Y. Patil Vidyapeeth's Dr. D. Y. Patil Dental College, Pimpri, Pune -411018, Maharashtra, India.

Correspondence: SPK, e-mail: <saurabh.kakade17@gmail.com>. Received 14 March 2013 and revised version accepted 23 October 2013. Competing interests: none declared. 
Table. Demographic and periodontal characteristics $(n=66)$

\begin{tabular}{lccc}
\hline & $\begin{array}{c}\text { Cases }(n=22) \\
\text { Mean (Standard } \\
\text { Deviation) }\end{array}$ & $\begin{array}{c}\text { Controls ( } n=44) \\
\text { Mean (Standard } \\
\text { Deviation) }\end{array}$ & $p$ value \\
\hline Age & $20.95(5.34)$ & $20.95(5.27)$ & - \\
Time since having T1DM (years) & $9.86(5.77)$ & - & $0.92(0.37)$ \\
Gingival index & $1.40(0.35)$ & $3.57(3.44)$ & $0.00^{*}$ \\
No. of bleeding sites & $12.32(7.03)$ & $0.66(1.32)$ & $0.00^{* * *}$ \\
No. of periodontal pockets & $2.233 .19)$ & $0.80(1.57)$ & $0.08^{* *}$ \\
No. of clinical attachment sites lost & $2.14(2.93)$ & & \\
\hline
\end{tabular}

* Mann Whitney U Test, ** Independent Sample t-Test

\section{Discussion}

The present study was undertaken in a single centre. The status of periodontal disease was assessed based on the presence and extent of gingival inflammation measured as bleeding on probing, pocket depth and clinical attachment loss. Clinical attachment loss is considered the gold standard measure of previous experience (for periodontitis) in contrast to current disease and activity. When compared with pocket depth, clinical attachment loss is considered to be a more accurate measure of history of the disease and disease progression [7].

The mean number of bleeding sites, gingival index score, number of periodontal pockets and number of clinical loss of attachment sites were all significantly more in cases than in the controls. This is similar to findings by other authors [8-10].

T1DM may increase the risk of destruction of the periodontium. Local immune responses may be altered in T1DM and the lesions produced by microbial agents associated with a lesser tissue repair capacity might explain the increased deterioration of periodontal structures observed in diabetics [9].

Longitudinal studies are needed to find the causal relationship and risk factors for periodontitis in T1DM. Maintenance of good oral hygiene should be emphasized for those with T1DM to prevent this complication.

\section{References}

1. American Dental Association. Diagnosis and Classification of Diabetes Mellitus. Diabetes Care 2011; 34: S62-9.
2. Chávarry NG, Vettore MV, Sansone C, Sheiham A. The relationship between diabetes mellitus and destructive periodontal disease: a meta-analysis. Oral Health Preventive Dentistry 2009; 7: 107-27.

3. Kalra S, Kalra B, Sharma A. Prevalence of type 1 diabetes mellitus in Karnal district, Haryana State, India. Diabetology and Metabolism Syndrome 2010; 2: 14

4. American Dental Association. Diagnosis and classification of diabetes mellitus. Diabetes Care 2005; 28: S37-42.

5. Löe H, Silness J. Periodontal disease in Pregnancy: 1. Prevalence and Severity. Acta Odontologica Scandinavica 1963; 21: 533-51.

6. World Health Organisation. Reliability and Validity of data. In: Oral Health Survey: Basic Methods 4th ed. Geneva: World Health Organisation, 1997; 13-5.

7. Page RC, Eke PI. Case definitions for use in populationbased surveillance of periodontitis. Journal of Periodontology 2007; 78: 1387-99.

8. Dakovic D, Pavlovic MD. Periodontal disease in children and adolescents with type 1 diabetes in Serbia. Journal of Periodontology 2008; 79: 987-92.

9. Silvestre FJ, Miralles L, Llambes F, Bautista D, SoláIzquierdo E, Hernández-Mijares A. Type 1diabetes mellitus and periodontal disease: relationship to different clinical variables. Medicina Oral Patologia Oral y Cirugia Bucal 2009; 14: E175- 9.

10. Miralles L, Silvestre FJ, Hernández-Mijares A, Bautista D, Liambes F, Grau D. Dental caries in type 1 diabetics: influence of systemic factors of the disease upon the development of dental caries. Medicina Oral Patologia Oraly Cirugia Bucal 2006; 11: 256-60. 